\title{
NEW THEORY TO SOLVE WINDING PROBLEM OF GALAXIES
}

\author{
Jayaram A $\mathbf{S}^{*}$. \\ Department of Mechanical Engineering, Associate Professor, Dr Ambedkar Institute of Technology \\ Bangalore-560056, India \\ E-mail: surya.jayaram@gmail.com
}

\begin{abstract}
:
There are many unsolved problems associated with spiral galaxies.One of the biggest unsolved problem of is winding problem. This paper aims at solving the problem by assuming a new theory. It is named as "Rolling theory of arms of spiral galaxies". It has been explained in detail, considering all the facts about the spiral galaxies. This theory is more appropriate than "standing wave theory". The paper also illustrates a possible reason for brief x-ray bursts found in many spiral galaxies. This paper also gives satisfactory solution to the constant velocity problem, without the need of dark matter. Thus it solves two of the problems associated with galaxies simultaneously.
\end{abstract}

Key words. Spiral galaxy,Stars, Winding problem,New theory, Rolling of arms, neutron stars.

\section{INTRODUCTION}

There are several types of galaxies. This paper focusses on one important type of Galaxy called "Spiral Galaxy". This type of galaxy is shown in figure-1.

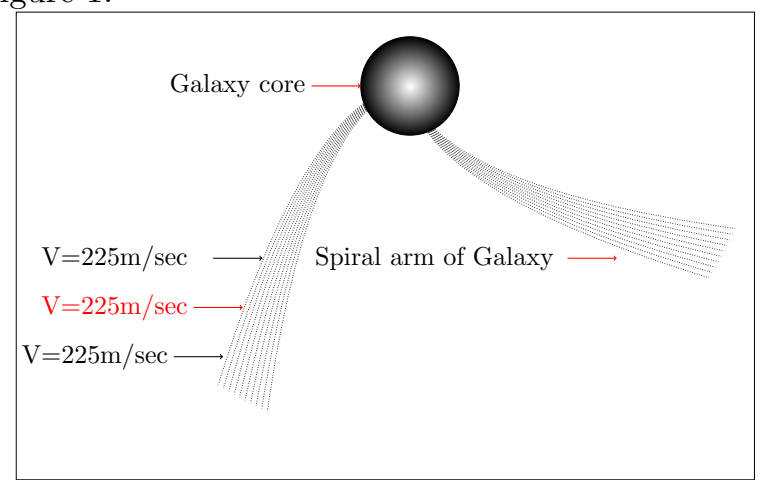

Fig. 1. Spiral Galaxy

The arms of the Spiral Galaxy consists of thousands of stars, revolving around the galaxy.All stars including the stars in the arms will rotate and revolve around the galaxy centre. By applying Newton's law of gravitation, we can calculate the speed at which each star should rotate for a stable orbit around the galaxy centre. As the distance increases velocity should also increase. In case of galaxies, stars will perfectly follow the law up to some distance of about 20000 light years. Beyond this distance, velocity of star is found to be almost constant. Although there is no perfect explanation for this, MOND theory explains it to a large extent. The constant velocity of all stars beyond 20000 light years is about $225 \mathrm{~km} / \mathrm{sec}$ in almost all galaxies. Sun is close to this distance and moving around our milky Way galaxy at about $222 \mathrm{~km} / \mathrm{sec}$. Since all stars beyond 20000 light years are moving at same velocity, the star 1 at 20000 light year away from the centre of galaxy should overtake another star 2, say at 21000 light years. Similarly, star 2 will overtake another star 3, little farther away at say 22000 light years. Due to this, after some thousands of years, the whole spiral arm should have to wind up as in 


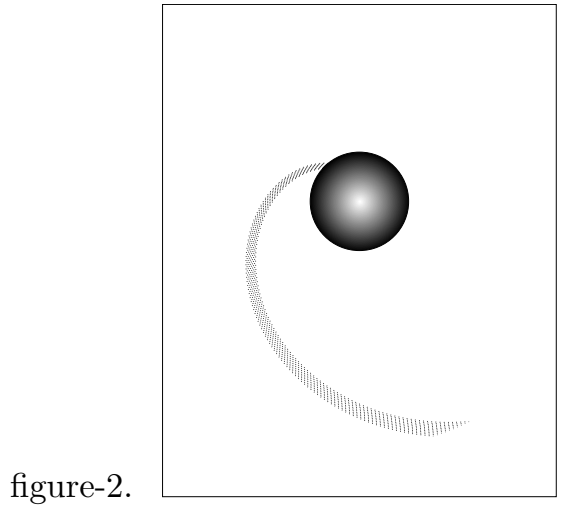

Fig. 2. Arm after winding up.

This is known as winding problem of Galaxies. We can find out thousands of old and new galaxies in the universe. But there is no evidence of already wound up Galaxy. Some thinkers are of the strange view that the star at the edge of the arm will somehow vanishes in to 4 th dimension and re appears in the next arm! So, there is no proper explanation for winding problem. This paper gives an explanation by assuming a rolling movements of stars.

Another problem associated with galaxy is the dark matter problem. Since the velocity of stars beyond 20000 light years is constant, it appears to violate Newton's law. If Newton's law is applied, then outer stars should fly away fro galaxies. But it is not happening. Therefore dark matter is assumed to surround the galaxy. That is still debatable. This rolling theory will solve that problem by assuming that radial velocity is appearing to be constant but it is having radial and tangential component. This makes the stars to obey Newton's law and Dark matter is not required to be present.

\section{ROLLING ARMS THEORY}

Main assumption of this theory is that stars in the arms of the spiral rolls in the circular path as shown in figure-3. It is like a pin wheel rotating with the axis at the centre. The line connecting the centre of the local disc and Galactic centre is the axis of rotation for the disc. In this rolling theory, it is assumed that because of the local gravity, the stars in the disc are rotating about local centre.

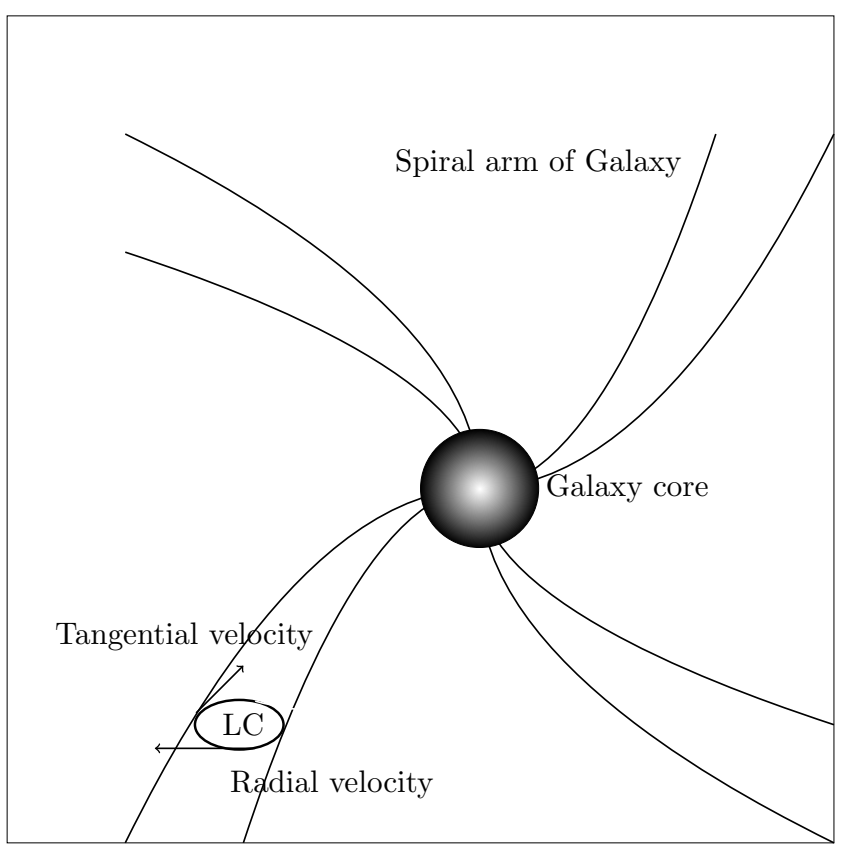

Fig. 3. Stars with different velocity components.

The stars at little closer to the centre of the galaxy will be rotating, having same tangential velocity but will be making more revolutions around the local centre. Thus, each star will be in the same arm almost at the same distance, avoiding winding of arms.

Even when the stars are moving in opposite direction, the relative velocity will be adequate velocity and thus avoiding falling into the center of galaxy. This can be realised from Sun-Earth-Moon system, as explained here. 


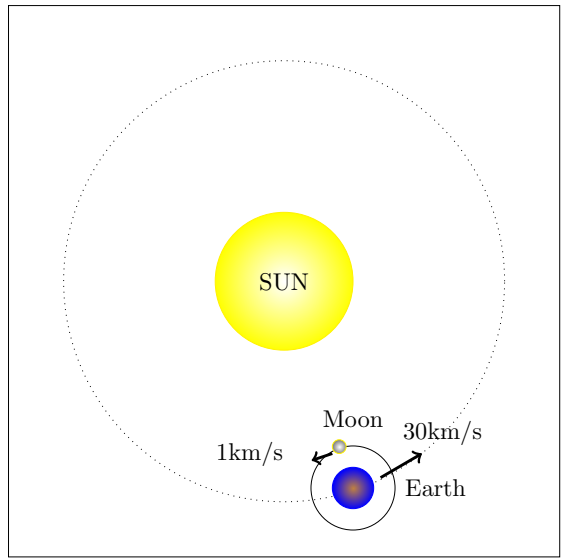

Fig. 4. Sun, Earth-Moon System

\subsection{Earth moon system}

As both earth and Moon are revolving around the Sun at about $30 \mathrm{~km} / \mathrm{sec}$ velocity, they are also revolving around their common centre at about $1 \mathrm{~km} /$ sec. So, any set of objects in the universe must be having a global centre and local centres. Sun is orbiting our Milky Way Galaxy, planets intern orbit the local centre , the Sun. Satellites orbit their local centre as Planets. The important point to be noted here is that even though it appears as if object like Moon to be moving in opposite direction as in fig-4, (earth-moon system)it will be having the base velocity of about $30 \mathrm{~km} / \mathrm{sec}$ around the Sun always and so it will be in its orbit around the Sun.

In case of Galaxy arms, local centres are neutron stars ( or black holes in some old galaxies ). So, local group of star system as a whole will be moving around the centre of the Galaxy at the required velocity but it is not the same star moving in same path in same speed. So, winding of arms will not happen.

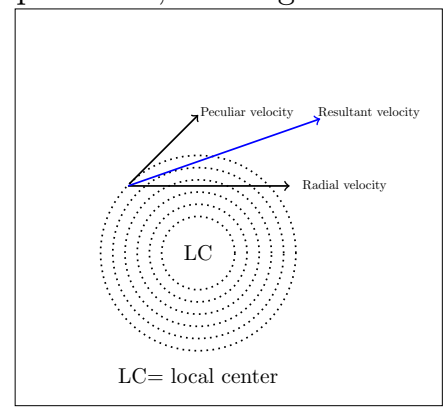

Fig. 5. Movement of stars around local centre

In every arm, the star in the outer most circle tends to move around the local centre. But due to the radial and tangential components of velocities as shown in figure-5, many stars tend to move in a direction closer to or away from the Galaxy centre. In some cases, many stars may move towards each other and collide. These are the effects of combined local and global gravity in the Galaxy. These collisions may lead to sudden release of large amount of energy, even as x ray bursts.

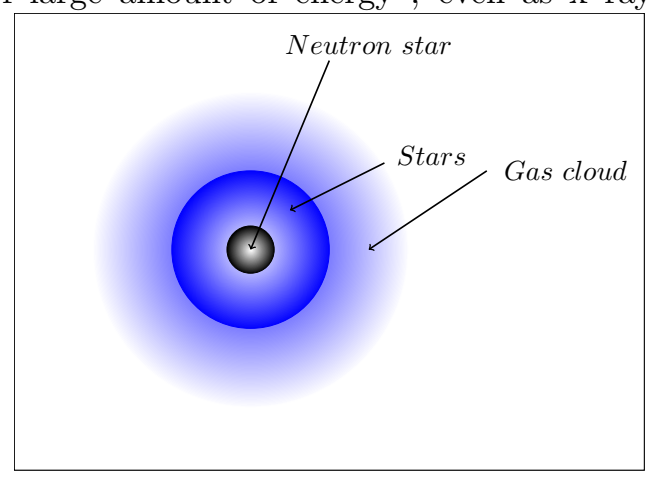

Fig. 6. Arrangement of stars according to density.

Arrangements of stars in arms and galaxies are obviously according to the density. Inner most stars in arms must be neutron stars and massive black holes will be at the centre of the Galaxy.So, the arrangement of stars are as shown in Fig-6.

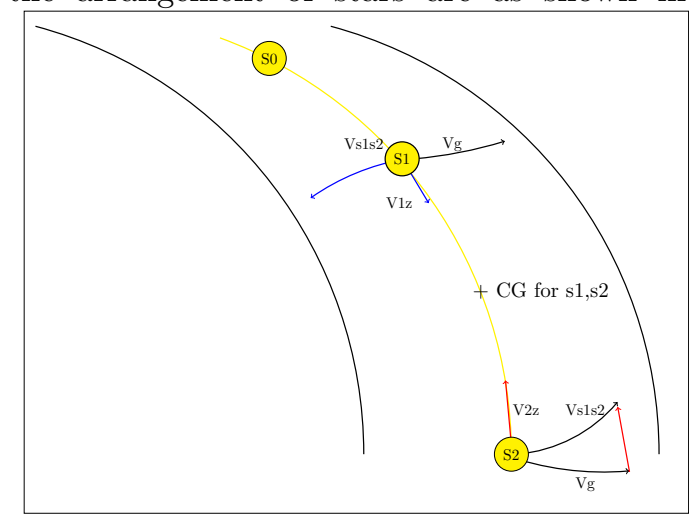

Fig. 7. Movement of stars towards centre of Galaxy.

Figure- 7 shows neutron stars S1, S2 at different distances in the arm of the galaxy. Here $\mathrm{S} 2$ is at farthest distance from the centre of the galaxy and S1 is little closer to the centre. Vg is the common notation used to represent the radial velocity component for both the stars. Since S1 and S2 are almost closer to each other, Vg is almost same for both. Assuming anticlockwise rotation of the galaxy, $\mathrm{Vg}$ is also anticlockwise. S1 will be nearer and tries to encircle the galaxy quicker but $\mathrm{S} 2$ will pull it back and similarly, $\mathrm{S} 2$ will be slower in angular velocity and so S1 will pull it forward. As a result, there will be a couple of forces at S1 and S2, shown by common symbol Vs1s2. Both stars tend to rotate around their common centre of gravity marked as CG of s1,s2, at the location shown by ?+? mark But for S2 will have $\mathrm{Vg}$ and Vs1s2 in almost same direction and added to give resultant component V2z. Similarly, another inner star will pull S1 towards the centre of the galaxy. 
But in S1, Vg and Vs1s2 are almost in opposite direction and so the resultant V1z towards S2 is much smaller than V2z. So, S2 moves towards the centre of the Galaxy at a faster rate than S1. Hence neutron stars S1 and S2 are likely to collide in this process. This is the most likely reason for $\mathrm{X}$-ray or fast radio bursts from galaxies.

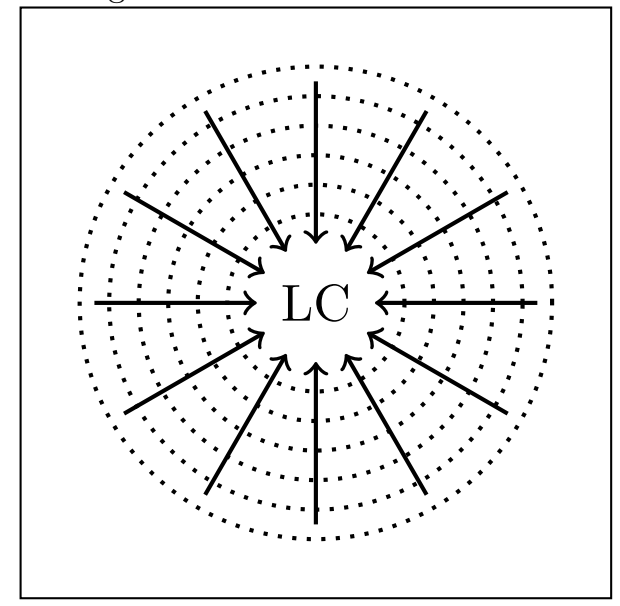

Fig. 8. Inward movement of stars.

Every star in the outer ring will try to occupy the position of local centre. By this also there will be collision of stars of almost neutron stars. After occupying the centre, it becomes more massive after several thousand years and it will also start moving towards the centre of the galaxy as explained before. This process will continue till the whole galaxy becomes a big black hole.

\section{CONCLUSIONS}

- This paper establishes a new theory of rolling of arms of Galaxy

- it clearly illustrates why standing wave theory appears to be true but not true

- it considers local gravity effect in addition to global gravity of the center of the Galaxy

- it gives most likely reason for x-ray and FRB emissions from distant galaxies

- it also establishes the reason for collisions of neutron stars in the arms of the Galaxy.

- it establishes "rolling arms theory" a better theory compared to standing wave theory.

\section{REFERENCES}

[1] Jesús Zavala $1,{ }^{*}$ OrcID andCarlos S. Frenk 2OrcID

Journals Galaxies Volume 7 Issue 4

[2] Rajendra P. Gupta

Journals Galaxies Volume 7 Issue 3

[3] Ranieri Diego Baldi 1,2, ${ }^{*}$ OrcID,Eleonora Torresi 3,et al, Journals Galaxies Volume 7 Issue 3 .

[4] Patrick M. Ogle1, Lauranne Lanz1, Cyril Nader1,2, and George Helou1, The Astrophysical Journal, Volume 817 , Number 2

[5] Victor P. Debattista 1 and J. A. Sellwood. The Astrophysical Journal, 513:L107-L110, 1999 March 10

[6] S. Mendoza, Canadian Journal of Physics, 2015, 93(2): 217-231

[7] Mordehai Milgrom, arXiv:1404.7661v2 [astroph.CO] 31 Aug 2014

[8]Benoit Famaey1 and Stacy McGaugh2 Journal of Physics: Conference Series, Volume 437, conference 1

[9] Jayaram.A.S, IJIFR/V4/ E2/ 048 Page No. 52565260

[10] Jayaram.A.S, vixra.org, volume 1 , issue 16120035 ,page 4. 